\title{
Experiência do Hospital Haroldo Juaçaba com Reconstrução Utilizando Retalhos Miocutâneos em Cirurgia para Tratamento do Câncer de Pênis locorregionalmente Avançado
} Experience of Reconstruction Using Myocutaneous Flaps in Surgery for Penile Cancer locoregionally Advanced Treatment at Haroldo Juaçaba Hospital Experiencia del Hospital Haroldo Juaçaba con Reconstrucción Utilizando Injertos Miocutáneos em Cirugía para Tratamiento del Cáncer de Pene loco regionalmente Avanzado

\author{
Márcio Alencar Barreira ${ }^{1}$ Lucas de Oliveira Lima ${ }^{2}$; Josualdo Justino Alves Júnior ; Lúcio Flávio Gonzaga Silva $;$ Marcos Venício Alves Lima ${ }^{5}$
}

\begin{abstract}
Resumo
Introdução: $\mathrm{O}$ Brasil é um país que apresenta uma alta incidência em câncer de pênis. Geralmente o diagnóstico dessa afecção é feito no curso inicial da doença, porém $1 / 3$ dos pacientes se apresentam com doença local ou regional avançada. Em casos onde ocorrem ressecçóes extensas com grande perda de substância, pode ser necessária a utilização de retalhos músculo cutâneos. Objetivo: Identificar quais os tipos de retalhos mais utilizados e as principais complicaçôes na reconstrução de defeitos complexos pós-cirurgia para câncer de pênis locorregionalmente avançado em um centro de referência brasileiro. Método: Estudo retrospectivo, observacional e descritivo. População de 243 pacientes atendidos com diagnóstico de câncer de pênis no Hospital Haroldo Juaçaba, entre janeiro de 2000 e setembro de 2010. Amostra de 35 pacientes que necessitaram de reconstrução com retalho muscular. Resultados: $\mathrm{O}$ retalho utilizando o músculo reto abdominal foi o mais usado $(57,2 \%)$, seguido pelo fáscia lata $(31,4 \%)$ e grácilis $(11,4 \%)$. As complicaçóes precoces mais comuns foram infecção de sítio cirúrgico $(37,1 \%)$ e deiscência parcial do retalho $(37,1 \%)$. As complicaçôes tardias mais comuns foram linfedema crônico (32,3\%) e edema de bolsa escrotal (29,4\%). Conclusão: O retalho miocutâneo do músculo reto abdominal foi o mais utilizado e mostrou-se seguro. Essa e as demais técnicas utilizadas resultaram em expressivas taxas de morbidade, porém sem comprometer a viabilidade do retalho.
\end{abstract}

Palavras-chave: Neoplasias Penianas-cirurgia; Retalhos Cirúrgicos; Reto do Abdome; Procedimentos Cirúrgicos Urológicos; Procedimentos Cirúrgicos Reconstrutivos

\footnotetext{
Instituição Vinculada: Hospital Haroldo Juaçaba/Instituto do Câncer do Ceará.

${ }^{1}$ Cirurgião-Oncológico. Hospital Haroldo Juaçaba. Instituto do Câncer do Ceará. Fortaleza (CE), Brasil. E-mail: marciofmj@msn.com.

${ }^{2}$ Acadêmico de Medicina da Universidade Federal do Ceará (UFC). Bolsista de Iniciação Científica do Instituto do Câncer do Ceará. Fortaleza (CE), Brasil. E-mail: lucaslimaufc@gmail.com.

${ }^{3}$ Médico e Mestre pela Universidade Estadual do Ceará. Ex-bolsista de iniciaçẫo Científica do Instituto do Câncer do Ceará. Fortaleza (CE), Brasil.E-mail: josujajr@gmail.com.

${ }^{4}$ Membro do Serviço de Uro-Oncologia do Hospital Haroldo Juaçaba e Chefe do serviço de Urologia da UFC. Mestre e Doutor pela UFC. Fortaleza (CE), Brasil. E-mail: gonzaga-silva@ufc.br.

${ }^{5}$ Cirurgião-Oncológico pelo Instituto Nacional de Câncer José Alencar Gomes da Silva (INCA). Coordenador de Ensino do Instituto do Câncer do Ceará. Mestre e Doutor pela UFC. Fortaleza (CE), Brasil. E-mail: marcosvalima@hotmail.com.

Endereço para correspondência: Instituto do Câncer do Ceará. Rua Papi Júnior, 1.222 - Rodolfo Teófilo. Fortaleza (CE), Brasil. CEP: 60430-230.
} 


\section{INTRODUÇÃO}

A incidência de câncer peniano varia geograficamente, sendo raro em países mais desenvolvidos. Nos Estados Unidos, a incidência varia de 0,3 a 1,8/100.000 habitantes. Uma incidência maior têm sido observada em países subdesenvolvidos, como Uganda (2,8/100.000 habitantes) e regiōes do Brasil (1,5 - 3,7/100.000 habitantes). A menor incidência relatada é em Israel $(0,1 / 100.000 \text { habitantes })^{1}$.

$\mathrm{O}$ câncer de pênis representa $2,1 \%$ das neoplasias masculinas do país, atingindo $5,7 \%$ no Nordeste. Não surpreendentemente, a região é responsável por mais da metade (53\%) dos casos registrados no Brasil. No Hospital Haroldo Juaçaba $(\mathrm{HHJ})$, são atendidos dois a três casos novos de câncer de pênis por mês ${ }^{2}$.

Os tumores metastáticos são incomuns e geralmente ocorrem tardiamente no curso da doença ${ }^{3}$. Apenas um terço dos pacientes se apresenta com doença local ou regional avançada e $10 \%$ dos pacientes inicialmente diagnosticados com doença localizada, posteriormente, apresentarão metástases a distância ${ }^{4}$.

A cirurgia é o método de escolha no tratamento do câncer de pênis invasivo. Em geral, a penectomia parcial é preferida nos indivíduos com doença T1 e em casos selecionados de $\mathrm{T} 2$ para manter margens histológicas de 1 $\mathrm{cm}$ (tumores bem e moderadamente diferenciados) a 1,5 $\mathrm{cm}$ (tumores pouco diferenciados). Para os pacientes com lesôes T2-4 ou naqueles T1 em que o resultado funcional e oncológico não seriam adequados, favorece-se a penectomia total ${ }^{5}$. A depender do caso, pode ser necessário realizar emasculaçáo e ressecção alargada.

No Hospital Haroldo Juaçaba, pacientes com neoplasia peniana que apresentam invasão proximal do corpo cavernoso e acometimento da pele do saco escrotal são submetidos rotineiramente à emasculação que inclui penectomia total, ressecção do saco escrotal e orquiectomia bilateral. Um trabalho que avaliou 16 pacientes submetidos a esse procedimento, em um período de dez anos, mostrou que a emasculação sem orquiectomia bilateral pode ser uma opção de tratamento segura, uma vez que apenas um dos 32 testículos clínicamente acometidos tinham infiltração focal microscópica do testículo ${ }^{6}$.

A linfadenectomia inguinal deve ser realizada em pacientes cN0 quando eles são considerados de alto risco para metástase oculta, ou seja, aqueles com tumores T2, G3, ou evidência de invasão linfovascular ${ }^{7}$. Torna-se ainda necessária, até mesmo como tratamento paliativo em alguns casos, pois resultam em feridas dolorosas e exsudativas.

Nas ressecções inguinais extensas, há grande perda de substância e, em algumas vezes, o fechamento primário não é possível. Dessa forma, se faz necessário o uso de retalhos miocutâneos, permitindo a cobertura de grandes defeitos de partes moles, reduzindo a morbidade do procedimento.

Indivíduos com envolvimento inguinal bilateral, disseminação extracapsular, linfonodos maiores de $2 \mathrm{~cm}$, envolvimento pélvico e mais de dois linfonodos envolvidos apresentam risco de morte que se aproxima de 50\% e parecem se beneficiar de quimioterapia adjuvante, porém é preciso mais estudos prospectivos e randomizados para definir melhor a população que mais se beneficiaria desse tratamento ${ }^{8}$.

O papel da radioterapia pós-operatória não é claramente definida na literatura, porém para doença linfonodal irressecável pode proporcionar paliação. Se a condição geral do paciente permitir, quimioterapia neoadjuvante pode tornar a doença linfonodal ressecável ${ }^{9}$.

O Ceará possui uma das mais altas incidências de câncer peniano do Brasil ${ }^{10}$, sendo o HHJ um centro de referência no tratamento dessa afecçáo. O objetivo deste trabalho é identificar quais os tipos de retalhos mais utilizados e as principais complicaçóes na reconstrução de defeitos complexos pós-cirurgia para câncer de pênis locorregionalmente avançado.

\section{MÉTODO}

Trata-se de um estudo retrospectivo, observacional e descritivo. A população estudada foi de 243 pacientes atendidos com diagnóstico de câncer de pênis, no HHJ, entre janeiro de 2000 e setembro de 2010, sendo que 210 pacientes foram submetidos a algum tipo de tratamento cirúrgico. Os outros 33 pacientes não tinham indicação cirúrgica por se apresentarem com doença avançada irressecável, metastática elou se encontrarem bastante debilitados. Aplicou-se a técnica amostral intencional, baseada em busca na base de dados do Registro de Câncer Hospitalar do $\mathrm{HHJ}$, bem como nos prontuários, tendo sido incluídos 35 pacientes que, devido à magnitude da ressecção, necessitaram de reconstrução com retalho miocutâneo.

Foi utilizado como instrumento de coleta de dados um formulário. As variáveis analisadas foram: tipos de técnicas cirúrgicas e complicaçóes pós-operatórias. Usaram-se retalhos com os seguintes músculos: reto abdominal, tensor da fáscia lata e grácilis. Não se baseou em uma classificação especifica. Convencionou-se que precoce seria a complicação ocorrida em até 30 dias da cirurgia com retalho e tardia as que aconteceram após essa data. Os dados foram armazenados e processados por meio do programa estatístico Statistical Package for the Social Science versão 18.0.

Este estudo foi aprovado pelo Comitê de Ética em Pesquisa do Instituto do Câncer do Ceará (ICC) / HHJ, sob o protocolo número 003/2010. 


\section{RESULTADOS}

Neste trabalho, 35 pacientes utilizaram algum tipo de retalho miocutâneo para reconstrução da perda tecidual após ressecção do câncer de pênis locorregionalmente avançado. A mediana de idade dos pacientes foi de 61 anos e o retalho utilizando o pedículo inferior do músculo reto abdominal foi o mais usado $(57,2 \%$ dos casos), como se pode verificar na Figura 1.

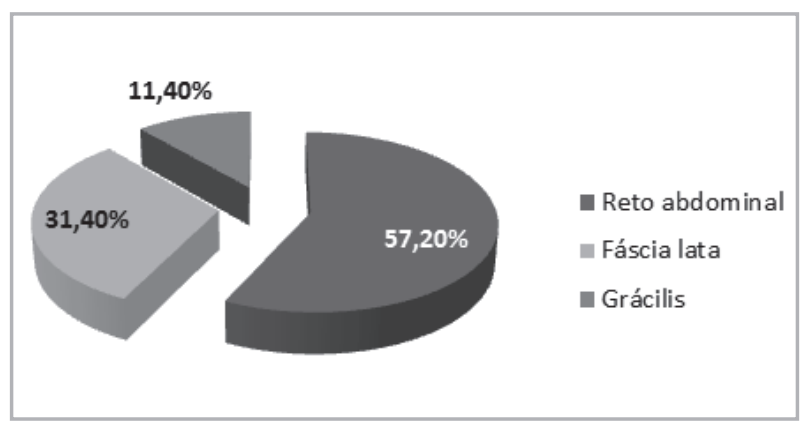

Figura 1. Distribuição de 35 pacientes submetidos à cirurgia para câncer peniano locorregionalmente avançado no Hospital Haroldo Juaçaba (Fortaleza, Ceará, Brasil) entre janeiro de 2000 e setembro de 2010, de acordo com o retalho miocutâneo utilizado

O retalho com músculo reto abdominal foi o que apresentou um menor tempo de internaçáo no pós-operatório. Isso fica bem evidente na Tabela 1 que também mostra que o retalho de músculo grácilis apresentou um maior tempo de internação hospitalar.
Tabela 1. Tempo de internação de 35 pacientes com câncer peniano locorregionalmente avançado submetidos à cirurgia, utilizando retalhos musculares no Hospital Haroldo Juaçaba (Fortaleza, Ceará, Brasil) entre janeiro de 2000 e setembro de 2010

\begin{tabular}{l|c|c|c}
\hline $\begin{array}{c}\text { Retalho } \\
\text { utilizado }\end{array}$ & $\begin{array}{c}\text { Tempo } \\
\text { mínimo } \\
\text { (dias) }\end{array}$ & $\begin{array}{c}\text { Tempo } \\
\text { máximo } \\
\text { (dias) }\end{array}$ & $\begin{array}{c}\text { MEDIANA } \\
\text { (dias) }\end{array}$ \\
\hline $\begin{array}{l}\text { Reto } \\
\text { abdominal }\end{array}$ & 12 & 68 & 18 \\
\hline Fáscia lata & 15 & 42 & 22 \\
\hline Grácilis & 9 & 49 & 26 \\
\hline
\end{tabular}

As complicaçôes precoces foram: infecção de sítio cirúrgico, epidermólise, necrose do retalho, deiscência de ferida ou retalho, linfocele, hematoma, seroma, trombose venosa profunda, abscesso pélvico, embolia pulmonar, pneumonia e infarto agudo do miocárdio. As complicaçôes tardias foram: linfedema crônico, trombose venosa profunda, erisipela, úlcera de membro inferior e edema da bolsa escrotal.

As complicaçôes precoces mais comuns foram infecção de sítio cirúrgico e deiscência de retalho, e as complicaçôes tardias mais comuns foram linfedema crônico dos membros inferiores e edema de bolsa escrotal (Tabela 2) que estão relacionados, principalmente, à linfadenectomia.

Houve um óbito $(2,8 \%)$. O paciente fez reconstrução utilizando retalho de músculo reto abdominal e faleceu devido a complicações que ocorreram em até 30 dias da cirurgia. Logo, as complicaçôes tardias só foram avaliadas em 34 pacientes.

Tabela 2. Complicações precoces e tardias em pacientes com câncer peniano locorregionalmente avançado submetidos à cirurgia utilizando retalhos musculares no Hospital Haroldo Juaçaba (Fortaleza, Ceará, Brasil) entre janeiro de 2000 e setembro de 2010

\begin{tabular}{|c|c|c|c|}
\hline Tipos & Complicações & $\begin{array}{l}\text { Número de } \\
\text { pacientes }\end{array}$ & $\begin{array}{c}\text { \% Em relação ao número } \\
\text { total de pacientes }\end{array}$ \\
\hline \multirow{10}{*}{$\begin{array}{l}\text { Precoces } \\
\text { (35 pacientes) }\end{array}$} & Infecção de sítio cirúrgico & 13 & $37,10 \%$ \\
\hline & Deiscência de retalho & 13 & $37,10 \%$ \\
\hline & Epidermólise & 8 & $22,80 \%$ \\
\hline & Necrose parcial de retalho & 8 & $22,80 \%$ \\
\hline & Abcesso pélvico & 4 & $11,40 \%$ \\
\hline & Linfocele & 3 & $8,50 \%$ \\
\hline & Hematoma & 2 & $5,70 \%$ \\
\hline & Pneumonia & 2 & $5,70 \%$ \\
\hline & TVP & 2 & $5,70 \%$ \\
\hline & Seroma & 1 & $2,80 \%$ \\
\hline \multirow{5}{*}{$\begin{array}{l}\text { Tardias } \\
\text { (34 Pacientes) }\end{array}$} & Linfedema crônico & 11 & $32,30 \%$ \\
\hline & Edema de bolsa escrotal & 10 & $29,40 \%$ \\
\hline & TVP & 5 & $14,70 \%$ \\
\hline & Úlcera de membro inferior & 2 & $5,80 \%$ \\
\hline & Erisipela & 1 & $2,90 \%$ \\
\hline
\end{tabular}

*TVP: Trombose Venosa Profunda. 
A Figura 2 mostra que o retalho de músculo reto abdominal foi o que apresentou menos complicaçóes e os outros retalhos tiveram um alto número de complicaçóes.

A Figura 3 apresenta um caso de recidiva inguinal unilateral extensa, com doença peniana controlada, necessitando para reparo uso da técnica do retalho de músculo reto abdominal.

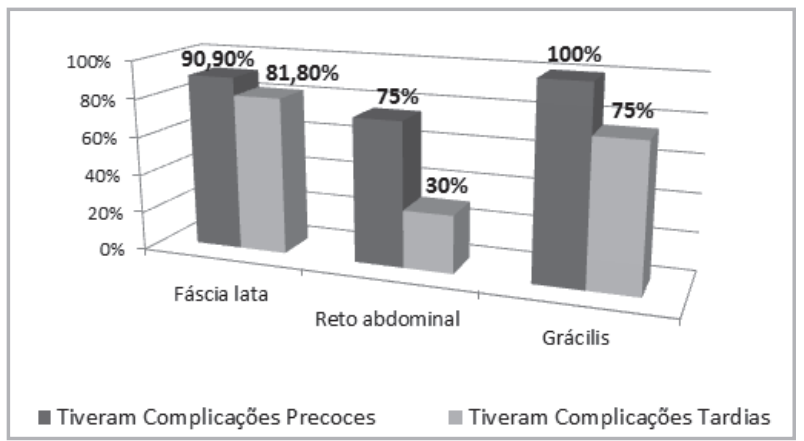

Figura 2. Incidência de complicações pós-operatórias precoce e tardia de acordo com o retalho miocutâneo utilizado em pacientes com câncer peniano locorregionalmente avançado, utilizando retalhos musculares no Hospital Haroldo Juaçaba (Fortaleza, Ceará, Brasil) entre janeiro de 2000 e setembro de 2010

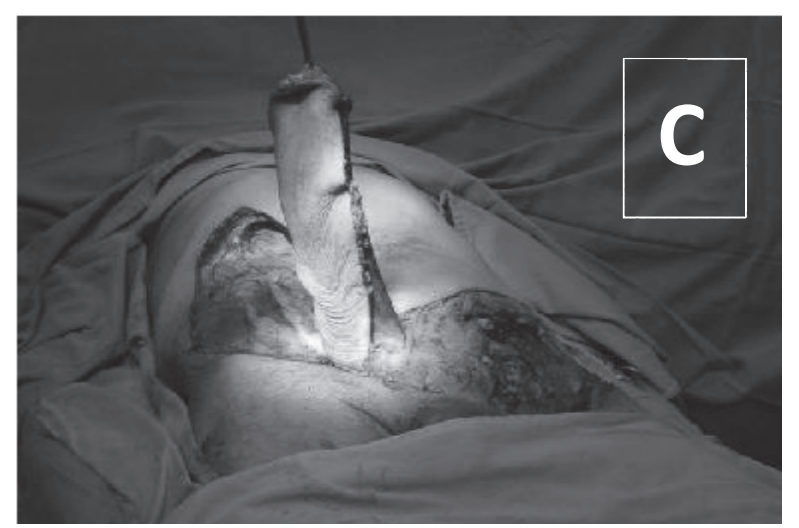

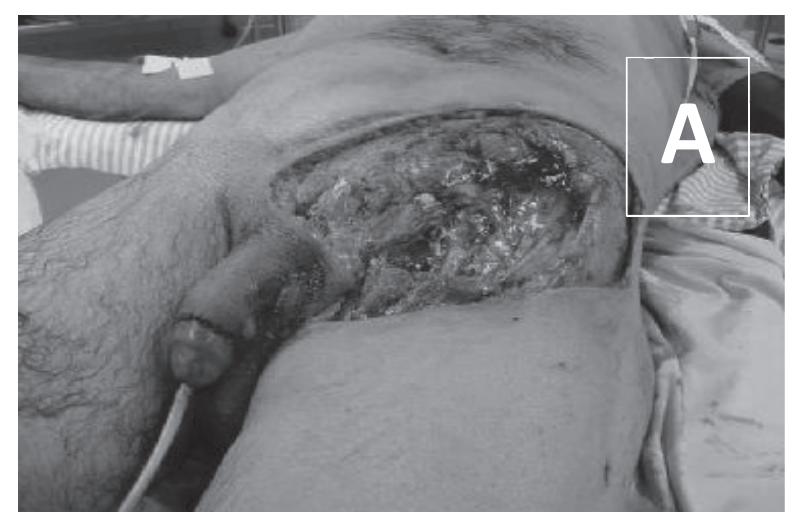

\section{DISCUSSÃO}

A utilização de técnicas restauradoras com retalhos miocutâneos se fez necessária em $17 \%$ dos pacientes operados com câncer de pênis no HHJ. O grande número de pacientes que necessitaram de cirurgia reconstrutora se deve ao diagnóstico tardio da doença e à recorrência da mesma. Na escolha do retalho, se levou em consideração a localização e complexidade do defeito, bem como as condiçóes locais da regiáo receptora.

O uso de retalhos musculares apresenta muitas vantagens. Sáo elas: reduzir espaço morto diminuindo a formação de seroma, fornecer um ambiente favorável à cicatrizaçáo de feridas por meio do uso de tecido bem vascularizado de uma área distante da virilha, oferecer proteção para os vasos femorais, reduzir a tensão durante o fechamento da ferida, iniciar a radioterapia mais precocemente com segurança e, ainda, pode encurtar a permanência hospitalar ${ }^{11}$.

O retalho mais utilizado foi o miocutâneo vertical do reto abdominal (VRAM) que é baseado no pedículo inferior do reto abdominal, portanto inverso ao da descriçâo original, em que se utiliza um retalho pediculado na artéria epigástrica superior ${ }^{12}$.
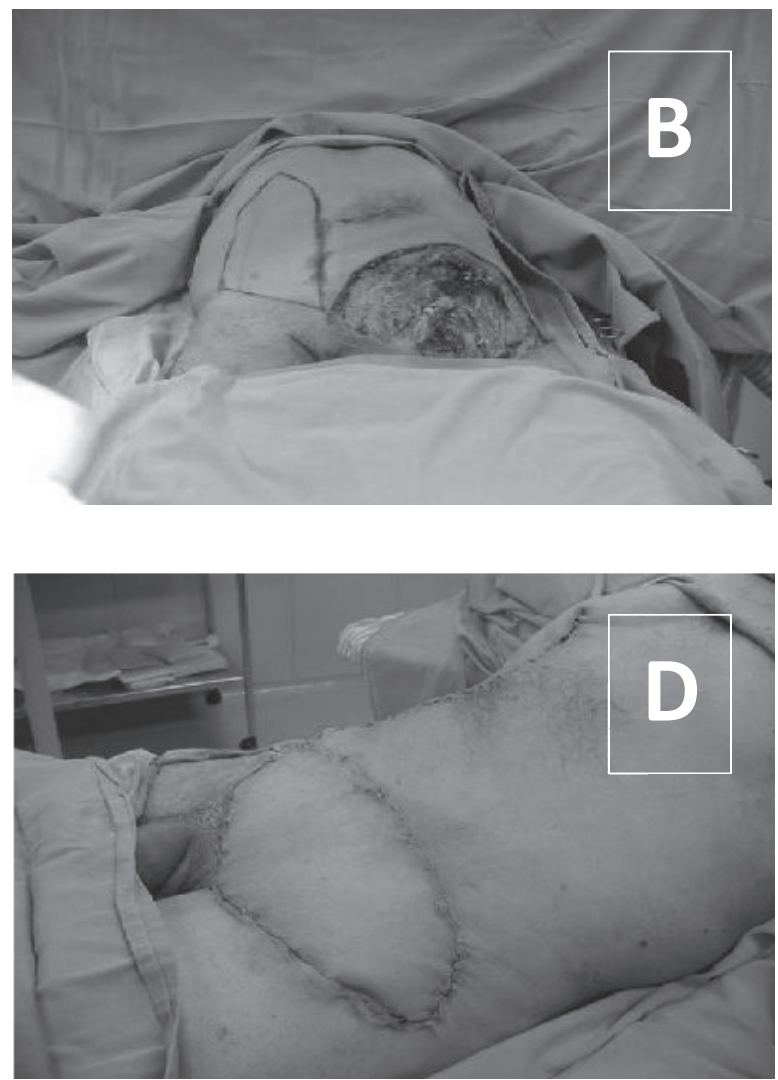

Figura 3. Paciente submetido à extensa ressecção de recorrência inguinal esquerda avançada de câncer de pênis e reparo do defeito com retalho miocutâneo vertical do músculo reto abdominal (VRAM) no Hospital Haroldo Juaçaba, Fortaleza, Ceará, Brasil. A. Aspecto da perda tecidual pós-ressecção; B. Demarcação da ilha de pele da área doadora; C. VRAM pronto para o uso; D. Resultado final

Fonte: Arquivo de imagens do Serviço de Urologia do Instituto do Câncer do Ceará. 
Algumas vantagens do VRAM são: vascularização segura resultando em baixos índices de perda do retalho, amplo arco de rotação, retalho volumoso com uma grande ilha de pele, local doador com morbidade aceitável e permite reconstruçóes do lado oposto quando os vasos epigástricos ipsilaterais forem ligados. Algumas das desvantagens e/ou complicaçôes desse tipo de retalho são: hérnia abdominal (9-17\% dos pacientes) e linfedema (até 32\% dos pacientes). A laparotomia exploradora ou cesárea anterior e obesidade mórbida são contraindicaçôes ao procedimento ${ }^{13-14}$.

Küntscher et al. ${ }^{15}$ analisaram os resultados do emprego do VRAM para cobertura de defeitos após ressecção de tumor peniano em sete pacientes e concluíram que esse retalho representa importante opção nesses casos. Kayes et al. ${ }^{16}$ estudaram quatro pacientes com doença metastática subcutânea por câncer avançado de pênis, submetidos à ressecção paliativa agressiva e à reconstrução com VRAM e não observaram complicações cirúrgicas.

Chessin et al. ${ }^{17}$ concluíram, em um trabalho realizado com 78 pacientes, submetidos à ressecção abdominoperineal após radio-quimioterapia para câncer de reto e canal anal, que o fechamento perineal com VRAM reduziu significativamente $(44,1 \%$ vs $15,8 \%)$ a incidência de complicaçôes da ferida perineal.

Buchel et al. ${ }^{18}$ discutiram as principais complicaçóes presentes no uso de retalhos VRAM para a reconstrução pélvica. Fizeram um trabalho retrospectivo com 73 pacientes submetidos a reconstruçôes pélvicas por patologias diversas, sendo a maioria carcinoma anorretal. Seus dados mostraram uma taxa de complicação de $22 \%$. Apesar dessas complicaçóes, a utilização de tecidos não irradiados tem um claro benefício na cicatrização de feridas. Este trabalho, também, mostrou que os pacientes que utilizaram o VRAM para a reconstrução inguinal apresentaram menores taxas de complicações em relação ao uso das outras técnicas de reconstrução.

O segundo retalho mais utilizado foi o tensor da fáscia lata (TFL) que tem como principais características a grande quantidade de pele disponível, grande arco de rotação e segurança do pedículo que se baseia na artéria femoral circunflexa lateral que tem seu trajeto entre os músculos reto femoral e vasto lateral ${ }^{19}$. Descrito pela primeira vez em 1934 por Wangensteen et al., foi inicialmente usado para as reconstruçóes da parede abdominal e mais tarde popularizado por Nahai et al. que introduziram muitas outras aplicaçôes (principalmente para reconstruir defeitos causados por úlceras de pressão e ressecçôes extensas na região pélvica ${ }^{20,21}$. Contudo, esse retalho não está isento de complicaçóes. Algumas delas não influenciam no resultado final e necessitam de reoperação pouquíssimas vezes. As taxas de complicações na área de reconstruçáo podem chegar até a $48 \%$, enquanto, na zona doadora, podem alcançar $18,5 \%{ }^{22}$. Nessa série, o TFL foi o segundo tipo de retalho mais utilizado e apresentou um grande número de complicaçôes precoces e tardias.

O retalho utilizando o músculo grácilis (RMG) foi o terceiro tipo de retalho mais utilizado e pode ser aplicado para reconstrução após ressecçôes inguinais extensas para câncer de pênis; porém é pouco usado. Ele proporciona adequada proteçáo tecidual, entretanto apresenta massa muscular pequena dificultando seu uso em lesóes mais extensas ${ }^{23}$.

Soper et al. ${ }^{24}$ concluíram que o VRAM substitui o RMG na reconstrução abdominoperineal e/ou exenteração pélvica. A comparação dos resultados da reconstrução pélvica com VRAM (114) e RMG (19) revelou significativamente maior incidência de complicaçōes ( $42 \%$ vs $15 \%$ ) no grupo que utilizou RMG.

Neste trabalho, também, foi observado que o RMG apresenta mais complicações do que o VRAM. Além disso, está relacionado a um maior tempo de internação hospitalar.

Uma das complicações mais frequentes, nas reconstruções com retalhos musculares, foi infecção de sítio cirúrgico $(37,1 \%)$; a literatura mostra que esse é o problema mais comum em pacientes cirúrgicos, sendo responsável por $40 \%$ das infecçóes hospitalares. A deiscência de ferida operatória $(37,1 \%)$ também foi uma complicação muito comum e costuma se desenvolver de 7 a 10 dias após a cirurgia, podendo ocorrer até 20 dias após o procedimento cirúrgico ${ }^{25}$. Essas complicaçôes foram tratadas conservadoramente com antibióticos e curativos, sem necessidade de cirurgia, na quase totalidade dos pacientes.

Já as complicaçôes tardias mais frequentes foram, de longe, linfedema crônico $(32,3 \%)$ e edema de bolsa escrotal $(29,4 \%)$. Estas relacionadas à linfadenectomia inguinal que ocasionou uma drenagem linfática prejudicada. $\mathrm{O}$ tratamento dessas complicaçóes foi, principalmente, com fisioterapia motora e sintomáticos.

Apenas um paciente faleceu até 30 dias da rotação do retalho. Trata-se de um idoso de 85 anos que adquiriu uma infecção respiratória que evoluiu para insuficiência respiratória e óbito após rotação de retalho utilizando músculo reto abdominal. Logo, esse paciente faleceu de uma causa não relacionada diretamente ao procedimento cirúrgico e sim a complicaçóes sistêmicas, possivelmente decorrente das comorbidades do paciente e do grande porte da cirurgia. Mostrando, dessa forma, que as cirurgias reconstrutoras estão relacionadas a uma baixa mortalidade.

\section{CONCLUSÃO}

O câncer de pênis avançado não possui uma opção terapêutica eficiente, sendo que a cirurgia, mesmo que paliativa, carece de retalhos miocutâneos para correçáo dos defeitos. O retalho do músculo reto abdominal foi o mais 
utilizado e mostrou-se seguro. Essa e as demais técnicas utilizadas resultaram em baixa mortalidade, apesar das expressivas taxas de morbidade, essas geralmente menores, sem que comprometessem a viabilidade do retalho.

As complicaçôes precoces mais comuns foram infecção de sítio cirúrgico e deiscência de retalho. As complicaçóes tardias mais comuns foram linfedema crônico e edema de bolsa escrotal.

\section{AGRADECIMENTOS}

À bibliotecária Cynthia Brasil e à Equipe de Cirurgia Plástica do ICC.

\section{CONTRIBUIÇÕES}

Todos os autores contribuíram na concepção e planejamento do estudo; na obtenção, análise e interpretação dos dados; e na redação e revisão crítica.

Declaraçáo de Conflito de Interesses: Nada a Declarar.

\section{REFERÊNCIAS}

1. Curado MP, Edwards B, Shin HR, Storm H, Ferlay $\mathrm{J}$, Heanue $\mathrm{M}$ et al., editors. Cancer incidence in five continents, Vol. IX. Lyon: IARC; 2007. (IARC Scientific Publications, 160)

2. Favorito LA, Nardi AC, Ronalsa M, Zequi SC, Sampaio FJ, Glina S. Epidemiologic study on penile câncer in Brazil. Int Braz J Urol. 2008; 34(5):587-91. Discussion 591-3.

3. Pow-Sang MR, Ferreira U, Pow-Sang JM, Nardi AC, Destefano V. Epidemiology and natural history of penile cancer. Urology. 2010; 76(2 Suppl 1):S2-6.

4. Culkin DJ, Beer TM. Advanced penile carcinoma. J Urol. 2003; 170(2 Pt 1):359-65.

5. Agrawal A, Pai D, Ananthakrishnan N, Smile SR, Ratnakar C. The histological extent of the local spread of carcinoma of the penis and its therapeutic implications. BJU Int. 2000; 85(3):299-301.

6. Gonzaga-Silva LF, Lima GR, Tavares JM, Pinheiro VO, Magalhães GA, Tomas ME, et al. Locally advanced penile carcinoma: classic emasculation or testis-sparing surgery?. Int Braz J Urol. 2012; 38(6):750-9.

7. Horenblas S. Lymphadenectomy for squamous cell carcinoma of the penis. Part 2: the role and technique of lymph node dissection. BJU Int. 2001; 88(5):473-83.

8. Katz A, Eifel PJ, Jhingran A, Levenback CF. The role of radiation therapy in preventing regional recurrences of invasive squamous cell carcinoma of the vulva. Int J Radiat Oncol Biol Phys. 2003; 57(2):409-18.

9. Singh I, Khaitan A. Current trends in the management of carcinoma penis: a review. Int Urol Nephrol. 2003; 35(2):215-25.
10. Sociedade Brasileira de Urologia. Primeiro estudo epidemiológico sobre o câncer de pênis [Internet]. 2007 [acesso 2 Jul. 2013]. Disponível em: http://www.sbu.org. br/pdf/bolu_232-2007.pdf

11. Murthy V, Gopinath KS. Reconstruction of groin defects following radical inguinal lymphadenectomy: an evidence based review. Indian J Surg Oncol. 2012; 3(2):130-8. Epub 2012 Mar 28.

12. Dinner MI, Labandter HP, Dowden RV. The role of the rectus abdomi-nismyocutaneous flap in breast reconstruction. Plast Reconstr Surg. 1982; 69(2):209-15.

13. Hoy E, Granick M, Benevenia J, Patterson F, Datiashvili $\mathrm{R}$, Bille B. Reconstruction of musculoskeletal defects following oncologic resection in 76patients. Ann Plast Surg. 2006; 57(2):190-4.

14. Lefevre JH, Parc Y, Kernéis S, Shields C, Touboul E, Chaouat $\mathrm{M}$, et al. Abdomino-perineal resection for anal cancer: impact of a vertical rectus abdominismyocutaneus flap on survival, recurrence, morbidity, and wound healing. Ann Surg. 2009; 250(5):707-11.

15. Küntscher MV, Mansouri S, Noack N, Hartmann B. Versatility of vertical rectus abdominismusculocutaneous flaps. Microsurgery. 2006; 26(5):363-9.

16. Kayes OJ, Durrant CA, Ralph D, Floyd D, Withey S, Minhas S. Vertical rectus abdominis flap reconstruction in patients with advanced penile squamous cell carcinoma. BJU Int. 2007; 99(1):37-40.

17. Chessin DB, Hartley J, Cohen AM, Mazumdar M, Cordeiro P, Disa J, et al. Rectus flap reconstruction decreases perineal wound complications after pelvic chemoradiation and surgery: a cohort study. Ann Surg Oncol. 2005; 12(2):104-10. Epub 2005 Feb 3.

18. Buchel EW, Finical S, Johnson C. Pelvic reconstruction using vertical rectus abdominismusculocutaneous flaps. Ann Plast Surg. 2004; 52(1):22-6.

19. Ramos RS, Cunha MS, Jesus RO, Basílio IA, Figuerêdo A, Fadul LC. Versatilidade do retalho musculocutâneo do tensor da fáscia lata. Rev Col Bras Cir. 2008; 35(3):155-8.

20. Wangensteen OH. Repair of recurrent and difficult hernias and other large defects of the abdominal wall employing the iliotibial tract of fascia lata as a pedicled flap. Surg Gynecol Obstet. 1934; 59:766-80.

21. Nahai F, Hill HL, Hester TR. Experiences with the tensor fascia lata flap. Plast Reconstr Surg. 1979; 63(6):788-99.

22. Williams JK, Carlson GW, deChalain T, Howell R, Coleman JJ. Role of tensor fascia lata in abdominal wall reconstruction. Plast Reconstr Surg. 1998; 101(3):713-8.

23. Mordjikian E, Oliveira MA, Merolo Júnior J. Métodos de reconstrução cirúrgica utilizados no tratamento das deformidades teciduais decorrentes da gangrena de 
Fournier: análise de 10 casos. Rev Soc Bras Cir Plást. 2001; 16(1):59-68.

24. Soper JT, Secord AA, Havrilesky LJ, Berchuck A, Clarke-Pearson DL. Comparison of gracilis and rectus abdominismyocutaneous flap neovaginal reconstruction performed during radical pelvic surgery:flap- specificmorbidity. Int J Gynecol Cancer. 2007; 17(1):298-303.

25. Townsend CM, Beauchamp RD, Evers BM, Mattox KL. Sabiston: tratado de cirurgia: a base biológica da prática cirúrgica moderna. 18. ed. Rio de Janeiro: Elsevier; 2009. p. 311. 


\section{Abstract}

Introduction: There is very high incidence of penile cancer in Brazil. Most patients are diagnosed early but one third present with advanced local or regional disease. In cases of major resection with substantial loss of tissue, musculocutaneous flaps may be necessary. Objective: to identify the most used types of flaps and their associated major complications in the reconstruction of complex defects following surgery for locoregionally advanced penile cancer at a Brazilian referral center. Method: A retrospective, observational and descriptive study involving 243 patients treated for penile cancer at Haroldo Juaçaba Hospital (Fortaleza, Brazil) between January 2000 and September 2010 of which 35 required reconstruction with surgical flaps. Results: The myocutaneous rectus abdominis flap was the most frequently used (57.2\%), followed by the fascia lata flap (31.4\%) and the gracilis flap (11.4\%). The most common early complications were infection of the surgical wound $(37.1 \%)$ and partial flap dehiscence $(37.1 \%)$. The most common late complications were chronic lymphedema (32.3\%) and scrotal swelling (29.4\%). Conclusion: The myocutaneous rectus abdominis flap was the most frequently used and was found to be safe. The techniques evaluated in this study were associated with significant levels of morbidity which did not compromise flap viability.

Key words: Penile Neoplasms-surgery; Surgical Flaps; Rectus Abdominis Urologic Surgical Procedures; Reconstructive Surgical Procedure

\section{Resumen}

Introducción: Brasil es un país que posee una alta incidencia de cáncer de pene. En general, el diagnóstico de esta enfermedad se realiza en su fase inicial, sin embargo, un tercio de los pacientes aparecen con la enfermedad, sea local o regional, en estado avanzado. En casos en que se verifican resecciones extensas, con pérdida significativa de substancia, puede ser necesaria la utilización de injertos musculo cutáneos. Objetivo: Verificar los tipos de injertos más utilizados y las principales complicaciones en la reconstrucción de defectos complejos post-quirúrgicos para cáncer de pene locorregionalmente avanzado en un centro de referencia en Brasil. Método: Estudio retrospectivo, observacional y descriptivo. Población de 243 pacientes atendidos con diagnóstico de cáncer de pene en el Hospital Haroldo Juaçaba, entre enero de 2000 y septiembre de 2010. Muestra de 35 pacientes que necesitaron reconstrucción con injerto muscular. Resultados: El injerto donde se utiliza el músculo recto abdominal fue el más empleado (57,2\% de los casos), seguido por el fascia lata $(31,4 \%)$ y grácil $(11,4 \%)$. Las complicaciones precoces más comunes fueron la infección de herida operatoria $(37,1 \%)$ y la dehiscencia parcial del injerto $(37,1 \%)$. Las complicaciones tardías más comunes fueron el linfedema crónico (32,3\%) y el edema de la bolsa escrotal (29,4\%). Conclusión: el injerto miocutáneo del músculo recto abdominal fue el más utilizado y se mostró seguro. Tanto ésta como las demás técnicas utilizadas tuvieron como resultado expresivas tasas de morbilidad, no obstante, sin comprometer la viabilidad del injerto.

Palabras clave: Neoplasias del Pene-cirurgía; Colgajos Quirúrgicos; Recto del Abdomen; Procedimientos Quirúrgicos Urológicos; Procedimientos Quirúrgicos Reconstructivos 